\title{
Research on Cognitive Matching of Biological Morphological Features and Images for Profiling Design
}

\author{
Bin Zhou and Li Lin* \\ School of Mechanical Engineering, Guizhou University, Guiyang, Guizhou, 550025, China
}

\begin{abstract}
To obtain high-quality bionic design scheme of product form, this paper explores the matching relationship between users' biological form features and their images from the level of implicit cognition, providing objective basis for effective selection of ideographic biological form features in bionic design of product form. The eye movement experiment was used to screen the biomorphic feature group that was focused on. Questionnaire survey and cluster analysis were used to obtain the main image phrases of the morphological feature group. The two collected materials were combined with implicit cognitive measurement (IAT) to obtain the response time data of the subjects in the classification task. According to Greenwald's method to verify the effectiveness of the data as a whole, the response time of the combination of various features and images in the compatibility group is sorted to obtain the design guidance conclusion. Taking the white shouldered eagle as an example, the experimental data showed high validity by t-test, and the implicit effect value of the compatibility group was 0.68 . According to the analysis of the data, the main image that most matches the head shape characteristics of the white shouldered eagle is "Ferocious", and the main image that most matches the wing shape characteristics is "Lightsome", and there is no difference in the implicit cognitive attitude between men and women. The designer takes this as the design reference to improve the effectiveness of the design output. This study can provide more objective suggestions for the bionic design of the related product shape.
\end{abstract}

\section{Introduction}

Nowadays, the bionic design of product shape can give the product a unique visual effect, making this kind of product more and more favored by consumers. The key of bionic design of product form lies in the effective extraction of biological form features and their representation images at the beginning of design [1] However, organisms can have a variety of perceptible images due to changes in external conditions. For example, cats can perceive images such as ferocity and flexibility when fighting and moving. Wrong extraction is easy to make users perceive the images expressed by products blurred [2] The biological morphological features extracted by designers only by experience and intuition inevitably lead to design failure due to lack of objective basis. Due to the separation of thinking and behavior, it is difficult to define whether the extraction results conform to the real cognitive situation of human heart only by seeking biological morphological features for copying design from eye movement tracks. Therefore, to obtain a design scheme that meets the users' inner real demands, it is urgent to explore the matching relationship between biological morphological characteristics and users' inner real image feelings from various implicit cognitive perspectives. Only in this way can we accurately select the biological morphological features that conform to the target image to carry out the design at the beginning of the copying design activities, thus reducing the waste of design resources and effectively improving the efficiency of product copying design.

\section{Implicit Cognition and Measurement}

The study of modern cognitive dual-processing theory shows that, Human cognitive model is divided into explicit cognitive process controlled by rational consciousness and implicit cognitive process controlled by intuition and unconsciousness [3] Conscious cognitive process is the premise of unconscious cognitive process. Unconscious cognitive process is the energy-saving mode of conscious cognitive process. Implicit cognitive process is more efficient because it is not controlled by consciousness, which greatly affects the judgment results made by users to the outside world [4]. General implicit cognitive measurement methods can objectively infer relevant cognitive results and attitudes by indirectly recording physiological data (such as eye movement trajectory, brain waves, reaction time, etc.) generated by the tester's implicit cognitive process [5]. Corresponding detection methods include eye movement tracking technology, EEG, Implicit Association Test (IAT) and Functional Magnetic Resonance Imaging (fMRI) [6]. Among them, IAT can infer the distance between the semantics of concepts and attributes on relevant nerves by indirectly measuring the matching reaction time of

"Corresponding author's e-mail: yangmq99@126.com 
concepts and attributes [7], effectively reducing the influence of impression management effect and reaction deviation on cognitive measurement results [8], and has certain efficiency and convenience. Therefore, using IAT measurement method to explore the image cognition situation automatically formed by users unconsciously from the perspective of implicit cognition will help to accurately express the meaning of copying design.

\section{Construction of Measurement Method Based on IAT}

Through the literature study of IAT paradigm, IAT paradigm can use words or pictures to represent the vast majority of concepts or attributes [9], as shown in Figure1.

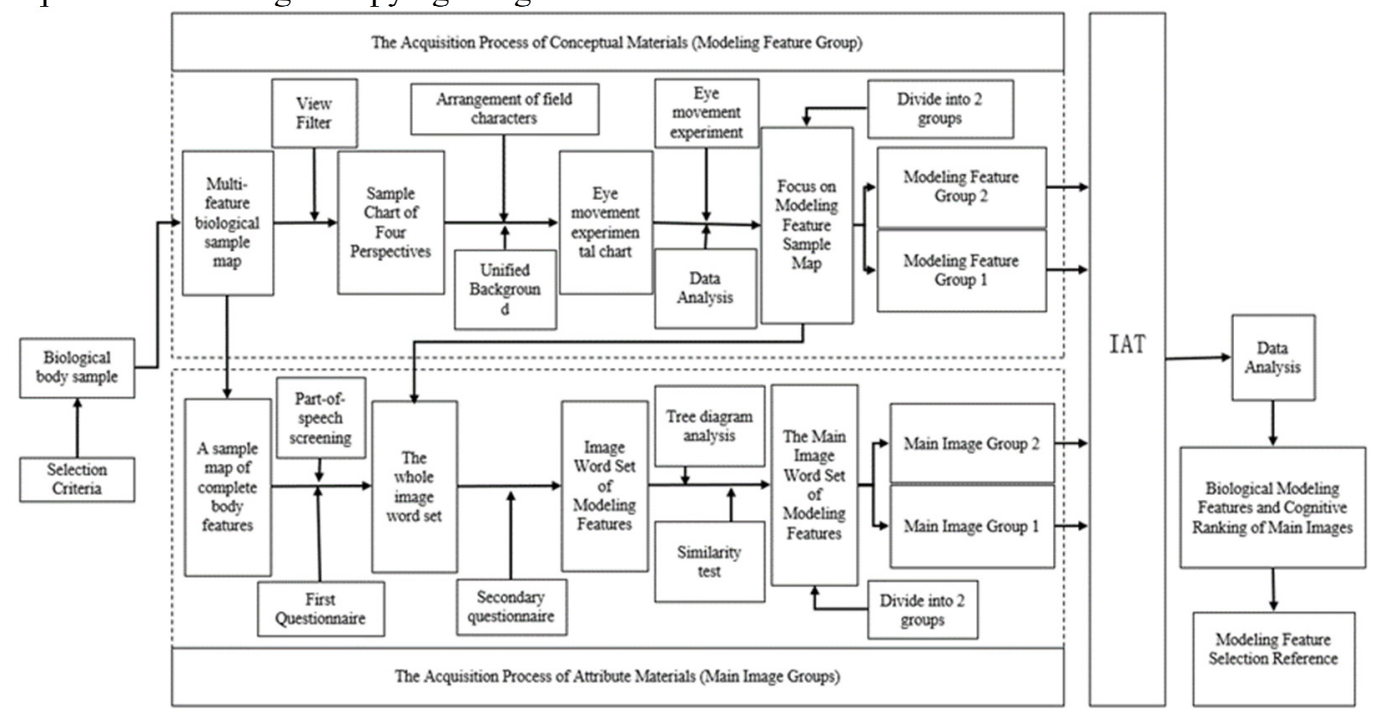

Fig 1. general flow of method

As shown in Figure1, This paper classifies the biological morphological features and the main images to which the features belong into the same category as the conceptual materials and attribute materials in IAT classical experiments. After combing the relevant literatures of IAT, Based on the analysis of the analogy between subjective images and objective things, this paper compares the different morphological features of organisms to "he" and "I" in conceptual materials, and compares the main images to which different morphological features belong to "positive words" and "negative words" in attribute materials, to construct an IAT-based measurement method.

\subsection{Preparation of IAT Test Materials}

As shown in FIG. 1, the experiment first collects complete body sample pictures of the target organism from various perspectives, and then carries out the following steps:

\subsubsection{Acquisition of conceptual materials (morphological characteristic group)}

First of all, the eye movement experiment material map adopts biological sample maps from any 4 viewing angles to be arranged in a field shape after being decolorized. According to the limited cognitive resources, people can rely more on the implicit association relationship in memory to make judgments [10], so the single set of the eye movement experimental maps only show 10 seconds, followed by 3 seconds of pure gray maps to eliminate the influence of the previous maps. Secondly, according to the biological topological structure analysis method [11], the individual morphological feature AOI (region of interest) of the biological subject in each group of the eye movement experimental maps is delineated for data statistics. The preferred morphological feature AOI data includes shorter first fixation time, longer fixation time and more fixation times [12]. Finally, according to the requirements of the IAT paradigm, 10 sample maps from different perspectives are selected from each of the top two morphological features as conceptual materials (morphological feature groups).

\subsubsection{Acquisition of attribute materials (main image groups)}

The whole image of the target creature was collected by a questionnaire survey with the complete body sample map, and the whole image word set of the creature was obtained after eliminating the inapplicable and repeated image words in the obtained results. The whole image word set and the morphological feature sample map extracted by eye movement were used for a secondary questionnaire survey to obtain the image word set of the morphological feature. Secondly, the words with check rate $\geq 30 \%$ are counted in the morphological feature image word set, the main image word set is screened out by similarity detection, and the results are analyzed in SPSS, and the tree diagram is obtained by Ward system clustering method, and the clustering number of images is determined according to the diagram. Finally, according to the requirements of IAT paradigm, the representative image words belonging to the first 10 clustering sets with high generality are taken as the main image word sets of the two morphological features, and 5 of the main image 
words are divided into main image group 1 and the rest are main image group 2.

\subsection{Cognitive Measurement and Analysis Based on IAT}

According to the classical IAT experimental paradigm and combined with the research content, the experimental steps are as follows:

\subsubsection{IAT experimental flow}

Based on E-prime 2.0 psychological experimental software, the materials obtained from the first two steps are programmed into IAT experimental program. The IAT lab procedure consists of seven steps, The first four steps are a set of experimental sections, In step 5, the combination of experimental material types is changed and the order of key reaction is reversed, 20 sample stimulus count are randomly displayed in each exercise step, The fourth and seventh steps are formal experimental data recording steps, showing a total of 40 sample stimuli, the specific experimental contents are displayed on the computer screen, the formal experiment is explained to the tester that this is a classified experiment to test the reaction speed, and 7 experimental steps are completed one by one according to the displayed instructions, Each display cycle first displays a $300 \mathrm{~ms}$ focus map to concentrate attention, then displays an unlimited key response map to randomly display morphological feature pictures or main image words. The top of the key response map has key prompts at all stages, while only a $100 \mathrm{~ms}$ feedback map will prompt right and wrong after the key is pressed in the practice stage.

\subsubsection{Experimental data processing}

According to the method given by GREENWALD [13], the data are imported into E-date for analysis. In the data, the reaction time below $300 \mathrm{~ms}$ is changed to $300 \mathrm{~ms}$, and the reaction time above $3000 \mathrm{~ms}$ is changed to $3000 \mathrm{~ms}$. The erroneous experimental results and the results with an error rate of more than $20 \%$ and the first two judgment reaction time data of each tester are eliminated. SPSS is used to count the average reaction time values of steps 4 and 7 in Table 1 respectively, and then the combination with lower reaction time values in all features and image combinations is identified as the compatible group. A paired t-test is carried out on the logarithmic values of the compatible group and incompatible group. If $\mathrm{P}<0.05$, the experimental results are statistically significant, indicating that the relationship between concepts and attributes in various combinations is established. The average reaction time of the male compatible group and the incompatible group is converted into logarithm and then the difference is taken (the data of female students are treated similarly). The logarithmic difference of reaction time between male and female is tested by a paired t-test. If $p>0.05$, the overall cognitive attitude is consistent.

\subsubsection{Matching Analysis of Features and Images}

According to the method described by Cai Huajian [14], the value at the time of conversion reaction is logarithmic, and the implicit effect $D$ value $(D=$ mean difference/standard deviation) is calculated as the effectiveness index to judge the overall measurement result. The implicit effect $\mathrm{D}$ values of the compatible group and the non-compatible group were calculated respectively, and the $\mathrm{D}$ value and 0 were tested by independent sample T-test. If the $\mathrm{D}$ value is greater than 0 at a certain significance level, it shows that there is a close connection between concepts and attributes (referring to morphological features and images in this experiment) in the original set groups, and the compatible group is more closely connected than the non-compatible group. According to the research of Li Yang et al. [15], if the implicit effect D value is positive, it indicates that the tester thinks that the compatible group is more closely matched. When the absolute value of $\mathrm{D}$ value is greater than 0.65 , it indicates that the combination has a strong IAT effect, indicating that the reaction of the combination can effectively reflect the cognitive situation of the tester. After the above verification, the cognitive ranking of features and images is obtained after arranging the reaction times of each group of compatible groups. Based on this, biological morphological features that take less time to match with images are selected as key ideographic features for relevant design reference.

\section{Instance Validation}

Taking the bird white-shouldered eagle as an example, the utility of the method proposed in this paper is verified.

\subsection{Preparation of Experimental Materials}

\subsubsection{Acquisition of conceptual materials (morphological characteristic group)}

The collected picture of the whole shape of the whiteshouldered eagle was screened by the above steps and used in the eye movement experiment. 20 college students (including 11 boys and 9 girls, aged 21-24) were taken as testers, and the eye movement data were imported into Be Gaze for analysis. In the eye movement experiment sample, the AOI region is divided according to the above method, and AOI 001 is set as wing, $\mathrm{AOI} 002$ is head, AOI 003 is Leg and claw, and AOI 004 is Tail. The AOI eye movement data of all trials are derived and sorted out statistically, as shown in Table 2. 
Table 2. test data of each AOI region

\begin{tabular}{ccccc}
\hline AOI Area & AOI 001 & AOI 002 & AOI 003 & AOI 004 \\
\hline Modeling Features & Wings & Head & Leg and claw & Tail \\
Average fixation duration/S & 3.35 & 3.66 & 2.33 & 0.66 \\
First fixation time/S & 0.54 & 0.36 & 0.71 & 0.87 \\
Fixation times & 5.98 & 6.29 & 3.58 & 2.39 \\
\hline
\end{tabular}

As can be seen from Table 2, the average fixation time of head morphological features is the longest, the first fixation time is the shortest, and the fixation times are the most, followed by wings. The graph groups of related features are sorted out, and the graph group of wing morphological features is set as the graph group of morphological features 1 , and the graph group of head morphological features is set as the graph group of morphological features 2 .

\subsubsection{Acquisition of attribute material (image group)}

The first image questionnaire was issued to obtain a total of 78 image words of White Shoulder Eagle, and the second image questionnaire counted 40 image words with a check rate of $\geq 30 \%$. A total of 110 questionnaires were collected from the similarity test questionnaire survey of morphological feature image word sets. There were 97 valid questionnaires, The effective rate was $97.67 \%$. SPSS 19.0 was used to cluster the questionnaire data by Ward system to obtain a tree diagram. The clustering number of image words was classified into 13 categories. Among the top 10 representative image words with more generality, " Highspeed, Flexible, Smooth, Lightsome, Swift" was set as the main image group 1, and "Grisliness, Powerful, Imposing, Ferocious, Intrepid" was set as the main image group 2 .

\subsection{IAT experimental measurement and analysis}

Forty college students were taken as IAT experimental testers, including 19 male students and 21 female students, aged 21-25 years old and right-handed. All the testers had not participated in similar related experiments before. The validity analysis of the obtained reaction time data was carried out. The specific steps are as follows:

\subsubsection{Determination of Compatible Groups}

After being processed by E-date software, the reaction time data distribution map of morphological features and main images in each group is obtained, as shown in Fig. 2.

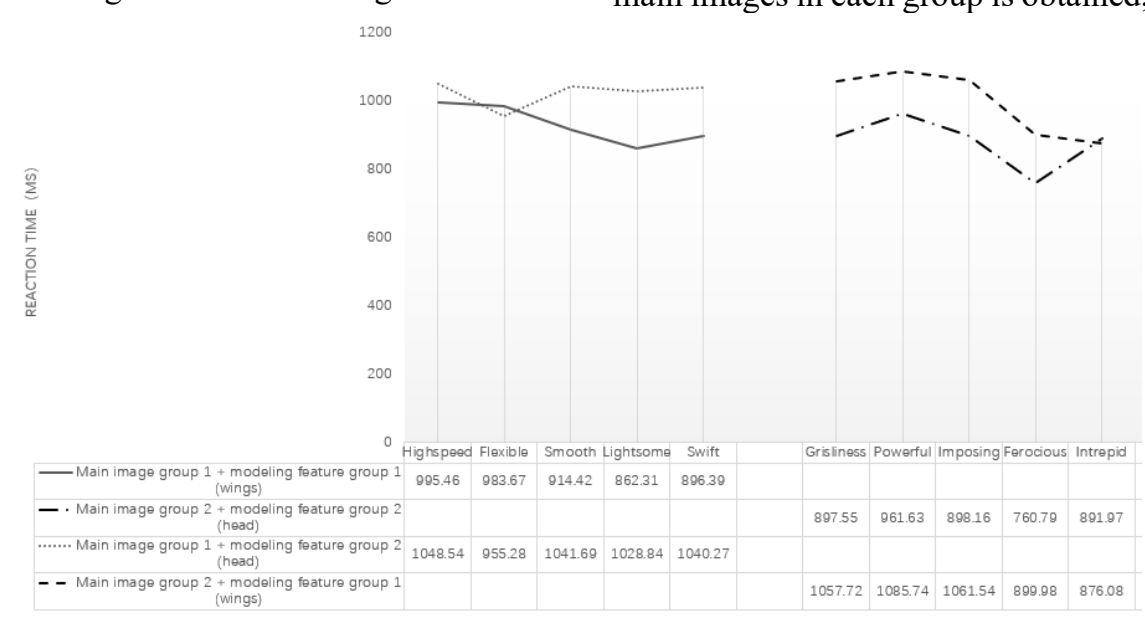

Fig 2. Reaction time data distribution map

Observing the distribution of reaction time numerical connection lines in FIG. 2, it can be seen that the average reaction time numerical connection lines of the main image group 1 and the morphological feature group 1 and the main image group 2 and the morphological feature group 2 are lower than other combinations on the whole, and can be determined as compatible groups, while the other combinations are incompatible groups.

\subsubsection{Analysis of the validity of reaction time data}

Paired T test was carried out on the logarithm of reaction time of compatible group and incompatible group, and the results are shown in Table 3. 
Tab 3. Each response numerical comparison

\begin{tabular}{ccccc}
\hline Group & $\begin{array}{c}\text { M (logarithm of } \\
\text { reaction time) }\end{array}$ & SD & $t$ & $\mathrm{p}$ \\
\hline Compatible group & 2.91 & 0.072 & -2.624 & 0.013 \\
Incompatible group & 2.98 & 0.099 & & \\
\hline
\end{tabular}

Table 3 shows that the obtained $\mathrm{p}<0.05$, and the test results are statistically significant, indicating that the connection between the features in various combinations and their images is established. As shown in Table 4, the reaction time paired $\mathrm{T}$ test for boys and girls by the above method is shown.

Tab 4. Cognitive differences between men and women

\begin{tabular}{ccccc}
\hline Gender & $\mathrm{M}$ & $\mathrm{SD}$ & $t$ & $\mathrm{p}$ \\
\hline Men & 0.68 & 0.31 & -0.32 & 0.09 \\
Women & 0.69 & 0.23 & & \\
\hline
\end{tabular}

As can be seen from table 4 , the obtained $p>0.05$ indicates that the overall cognitive attitude is consistent.
Calculate the implicit effect $\mathrm{D}$ value of each group and the independent sample $\mathrm{T}$ test results, as shown in Table 5.

Tab 5. D value and $\mathrm{T}$ test results of each group

\begin{tabular}{clccc}
\hline Group & D & SD & $t$ & $\mathrm{p}$ \\
\hline Compatible group & 0.68 & 1.543 & 2.624 & 0.013 \\
Incompatible group & 0.49 & 1.116 & 2.624 & 0.013 \\
\hline
\end{tabular}

As can be seen from Table 5, D values of both groups were greater than 0 , It is proved that the relationship between morphological features and main images in compatible and incompatible groups is valid. Also, the D values of the incompatible group and the compatible group are both positive, and the $\mathrm{D}$ values of the compatible group are greater than 0.65 . The independent sample $T$ test results of the two groups of $\mathrm{D}$ values and 0 show $\mathrm{P}<0.05$, which indicates that the test results are statistically significant, proving that the overall reaction time data can effectively reflect the real cognitive situation of the tester.

\subsubsection{Experimental conclusion}

After testing the validity of response time data, the analysis data shows that most of the testers subconsciously tend to associate the head shape characteristics with the image " Ferocious ", the wing characteristics with the image "Lightsome", and the matching cognitive attitude does not show a significant difference in gender. The analysis data shows that the implicit effect $D$ value of the compatibility group is 0.68 , indicating that the compatibility group The overall response time data can effectively reflect the matching cognitive status of morphological characteristics and main images of white shouldered eagles. Combined with FIG. 2, it can be seen that the main image with the shortest matching time with the head morphological features of the white-shouldered eagle is "Ferocious " and the main image with the shortest matching time with the wing morphological features is " Lightsome ", which shows that most testers truly agree with the matching relationship between "head- Ferocious " and "wing- Lightsome " of the white-shouldered eagle.
Therefore, when designers need to express the modelling design of light images, The wing morphological features of the white-shouldered eagle can be selected as the design emphasis. Similarly, when the modeling design needs to express fierce images, the head morphological features of the white-shouldered eagle can be analyzed and redesigned, to obtain the design output meeting the needs, which can indirectly prove that the method has certain practicability and guidance.

\section{Conclusion}

This paper is the first time to combine survey statistics and eye tracking with implicit association test, Obtaining the user's implicit reaction time data about the biological morphological features and the main images, Seeking the cognitive ranking of morphological features and main images, to assist designers to locate the key ideographic features of organisms at the beginning of design and obtain a copying design scheme that conforms to users' internal real cognition, can enhance the effectiveness of design output and has certain design guiding significance. Besides, this study uses implicit association detection method to make an exploration in the research field of biological design feature extraction, which has certain reference value for the same type of research. Besides, this paper explores the design-related research driven by objective data, based on reducing the influence of subjective factors on the design results, and can also provide some reference for the quantification and analysis of hidden needs within users. 


\section{References}

1. Luo Shi-jian, Zhang Yu-fei, BIAN Ze, et al. (2018). Research status and progress of bionic design for product shape. Journal of Mechanical Engineering, 54 (21) : 138155.

2. Zhang Xiang-quan. (2006). Research on bionic design of product morphology. Cha-ngsha: Hunan University.

3. MENG Ying-fang, GUO Chun-yan. (2006).Brain mechanism separation of implicit and explicit memory: an ERP study on face recognition. Journal of Psychology, (01):15-21.

4. RAND G, GREENE D, NOWAK A. (2012). Spontaneous Giving and Calculated Greed. Nature, (9): $427-430$.

5. Jiao Guangxia. (2010). Design and Research of Agricultural Vehicles Based on Kansei Engineering. Shandong University.

6. XIE Wei, XIN Xiang-yang, Hu Wei-feng. (2015). Research status of implicit measurement methods for users in product design. Journal of Mechanical Engineering, 32(02):105.

7. BRUNEL, F, TIE, B.C, \& Greenwald, A.G. (2004). Is the Implicit Association Test a valid and valuable measure of implicit consumer social cognition?. Journal of Consumer Psychology, 14:385-404.

8. CAI Hua-jian. (2003). effect of implicit self-esteem and the relationship between implicit self-esteem and explicit self-esteem. Journal of psychology, (06):796801.

9. Fu Ya-nan. (2018). Influence of warning pictures on heroin abusers' implicit attitude-s: Lanzhou: Northwest Normal University, 2018.

10. HAO Hong-da, WANG Yong. (2013). Implicit association test and consumer psychology. Advances In Psychological Science, (10): 1865-1873.

11. Xu Hong-lei. (2015). Research on bionic design of product form under the constraint of topological properties. Wuxi: Jiangnan university.

12. TIAN Shao-xu, SHEN Pin-pin, GUO Yu-zhu. (2015). Color emotion research based on eye movement tracking technology. Journal of Communication University of China, (6):70-76.

13. A.G.GREENWALD, D.E.MCGHEE, J.L.K.SCHWARTZ. (1998). Measuring Individua-l Differences in Implicit Cognition: The Implicit Association Test. Journal of Personality and Social Psychology, 74: 181-198.

14. CAI Hua-jian. (2003). Introduction to implicit association test proposed by GREENWALD. Advances In Psychological Science, 11 (3) :339-344.

15. LI Yang, FU Guo-qun. (2018). A study on stereotypes of China's post-80s and post-90s generation based on IAT. Management Review, 30(08):138-152. 